\title{
Clinical Evaluation of the Effectiveness and Safety of «BIOMED WHITE COMPLEX/CHARCOAL» Dentifrice
}

Chirkova NV*, Popova TA and Shelkovnikova SG The Department of Faculty Dentistry, Voronezh N.N. Burdenko State Medical University, Russia *Correspondling author: Chirkova NV, The Department of Faculty Dentistry, Voronezh N.N. Burdenko State Medical University, Russia

Received: November 12, 2018; Accepted: December 19, 2018; Published: December 26, 2018

\begin{abstract}
To test effectiveness and safety of whitening toothpaste with charcoal, «BIOMED WHITE COMPLEX/CHARCOAL».
\end{abstract}

Open non-comparative study in 1 group. Comparison was made using initial parameters. 60 volunteers at the age of 19-25 participated in the research. Measuring of dental plague was done using Fedorov-Volodkina Index. Measuring of teeth sensitivity was done using Schiff sensitivity score and Shtorina Index. Rating of teeth color and whitening property of testing products was proven using method of comparing results which were achieved via scale Vita Classic. Remineralization of enamel was studied through test of enamel resistance. Anticavity effect was identified by Decay-Missing-Filled Index (DMF).

Toothpaste «BIOMED WHITE COMPLEX/CHARCOAL» showed good cleaning results after first usage - lowering of hygiene index was up to $17.2 \%$. It was found out that toothpaste possesses excellent remineralization effect. Usage of this toothpaste lowers cavities in a short term period (2 month). During the research no negative side effects were observed on any of the volunteers.

Keywords: Clinical Trials; Tooth; Toothpaste; Anti-Caries Effect; Remineralization; «BIOMED WHITE COMPLEX/CHARCOAL»

\section{Introduction}

Toothpastes that contain charcoal are new comers in oral health care market. Historically, charcoal was one of few materials used for teeth cleaning. First registrated usage of charcoal as a tool for oral health care was assigned to Hippocrates in ancient Greece [1]. Unlike other materials such as salt, charcoal does not damage enamel and has an advantage-ability to control halitosis signs. Charcoal is especially when activated has great absorbing effect. In early 2000-s worldwide health organization included activated charcoal as a main treatment for poisoning and overdosing [2-4]. Activated charcoal became a very popular medicine to combat toxins and chemical substances.

In some countries of South-West Asia charcoal was added to toothbrushes for achieving black color. It was stated that blending charcoal and nylon bristle helps lower halitosis, lower teeth plague, and also prevent bacteria growth which can grow within your toothbrush. However, these statements are not supported by any scientific research [5].

Coal is one of the components of some toothpastes, however the shape and composition of the charcoal, along with the particle size of its particles, can make it abrasive, so the roughness of the enamel surface is expected to increase [6]. Toothpastes which are considered ideal, - are those that can clean teeth while not making them more rough.

Usage of charcoal for teeth cleaning is practiced in many countries in the world including Great Britain, Italy, Cameroon, Nigeria, Tanzania, Senegal Republic, Bangladesh, India, and Malesia. Despite the growing interest from commercial companies to coal as a tool for oral health care, data from clinical studies is still lacking and existing proves are insufficient.

The main purpose of the study is a clinical assessment of cleaning effectiveness and safety of complex toothpaste with coal «BIOMED WHITE COMPLEX/CHARCOAL» including influence of the toothpaste on anti-cavity resistibility and enamel remineralization.

\section{Materials and Methods}

\section{Study design}

Open non-comparative study in 1 group. Comparison was made using initial parameters. 60 volunteers participated in the trial. All patients were given toothbrush «Splat Professional Complete» of medium hardness and toothpaste «BIOMED WHITE COMPLEX/ CHARCOAL». Patients were prohibited to use any mouthwashes or other toothbrushes and toothpastes.

Volunteers were using tested product for 2 minutes. Hygiene index was measured before and after the usage. Then patients were using the product 2 times a day for 2 minutes in the morning and evening for 8 weeks with visits to dentist on $28^{\text {th }}$ day of research (visit 2) and on 56th day of research (visit 3).

Assessment of teeth plague was done using Fedorov-Volodkina Index on Visit 1 before and after teeth cleaning for 2 minutes and also on Visits 2 and 3. Measuring of teeth sensitivity was done using Schiff sensitivity score and Shtorina Index on Visits 1, 2 and 3.

Dentin sensitivity to air and cold stimuli (probe Schiff) was determined by reaction of the patient on stream of compressed air, that is pointed at the tested surface (on pre-neck area from vestibular side of the teeth) at the distance near $1 \mathrm{~cm}$. Temperature of air stream was $19-21^{\circ}$ Cand impact was done during 1 second time using 


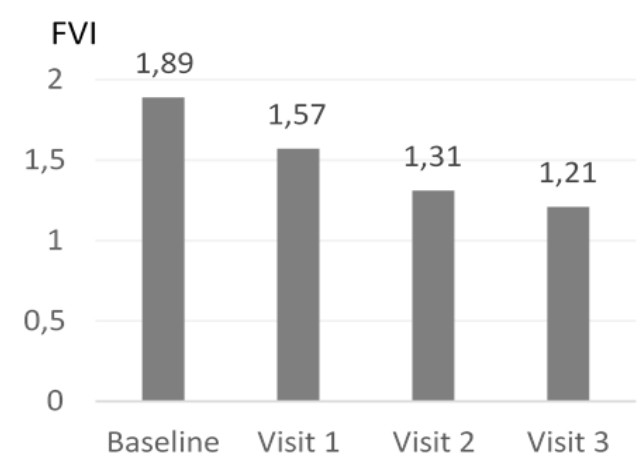

Figure 1: Assessment of the hygiene index.

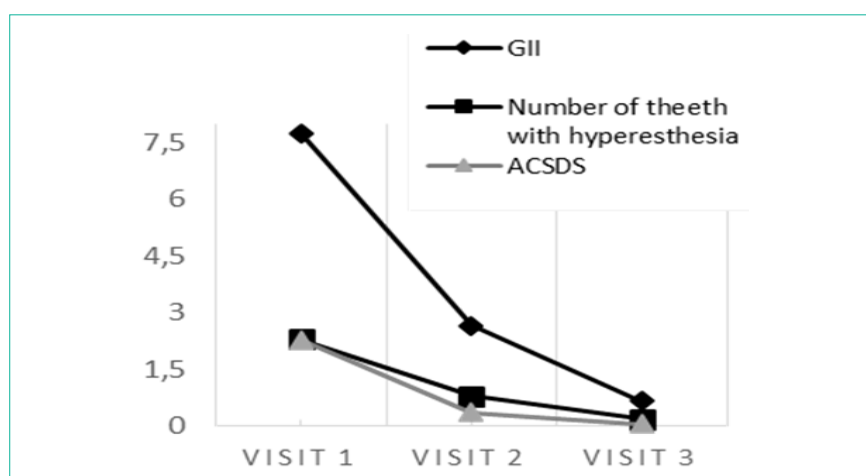

Figure 2: Comparison of mean changes in clinical parameters at baseline (visit 1), visit 2 and visit 3.

standard Air/water syringe of stomatological chair.

Visual analogue scale was proposed for self-assessment of pain levels of the patient on the probe. Assessment of teeth color and whitening property of tested products was done by method of comparing the results using Vita Classic scale obtained on visits 1 and 3 .

Assessment of enamel remineralization was done by using Test of Enamel Resistance (TER). The procedure was done on Visits 1 and 3. Anti-cavity effect was identified by Decay-Missing-Filled Index (DMF) during Visits 1 and 3.

\section{Patient selection criteria}

\section{Inclusion criteria were:}

1. Volunteers at the age from 18 to 50 years.

2. Amount of teeth not lower than 20.

3. Dark enamel via scale Vita-A4, B4, C4 measured at Visit 1.

\section{Exclusion criteria were:}

1. Professional teeth cleaning or teeth whitening were less than 90 days before the study according to anamnesis;

2. Any other dental manipulation including surgical and orthodontic in less than 30 days before the research according to anamnesis;

3. Any other condition or diseases that could prevent patient participation in the research or may interfere assessment of
Table 1: Characteristics of the subjects.

\begin{tabular}{|c|c|c|c|}
\hline \multirow{2}{*}{ Group } & Number of subjects & \multicolumn{2}{|c|}{ Age } \\
\cline { 3 - 4 } & 24 & Mean (士SD) & Range \\
\hline Men & 36 & $20.1( \pm 0.53)$ & $19-25$ \\
\hline Women & $20.1( \pm 1.09)$ & $19-21$ \\
\hline
\end{tabular}

Table 2: Assessment of the hygiene index.

\begin{tabular}{|c|c|c|c|c|c|}
\hline \multirow{2}{*}{ Index } & \multicolumn{2}{|c|}{ Visit 1 } & Visit 2 & Visit 3 \\
\cline { 3 - 6 } & $\begin{array}{c}\text { Pre-Brushing } \\
\text { (Baseline) }\end{array}$ & $\begin{array}{c}\text { Post- } \\
\text { Brushing }\end{array}$ & & \\
\hline $\mathrm{FVI}^{\mathrm{a}}$ & Mean $( \pm \mathrm{SD})$ & $1.89( \pm 0.33)$ & $1.57( \pm 0.23)$ & $1.31( \pm 0.17)$ & $1.21( \pm 0.12)$ \\
\hline $\mathrm{a} F \mathrm{FVI}=$ Fedorov-Volodkina Index & & &
\end{tabular}

Table 3: Evaluation of the desensitizing action mean ( $\pm S D)$.

\begin{tabular}{|c|c|c|c|}
\hline Index & Visit 1 & Visit 2 & Visit 3 \\
\hline GII $^{\mathrm{a}}$ & $7.75( \pm 2.39)$ & $2.66( \pm 2.09)$ & $0.67( \pm 1.41)$ \\
\hline $\begin{array}{c}\text { Number of teeth with } \\
\text { hyperesthesia }\end{array}$ & $2.3( \pm 0.67)$ & $0.8( \pm 0.63)$ & $0.2( \pm 0.42)$ \\
\hline ACSDS $^{b}$ & $2.28( \pm 0.42)$ & $0.37( \pm 0.31)$ & $0.07( \pm 0.14)$ \\
\hline
\end{tabular}

${ }^{\mathrm{a}} \mathrm{GI}=$ Gipertensia Intensity Index (Shtorina index)

${ }^{\mathrm{b}} \mathrm{ACSDS}=$ Air-Cold Stimulus Dentin Sensitivity (Schiff test)

researchable parameters according anamnesis's data and medical documentation;

4. Hypersensitivity to components of tested products or severe food allergy;

5. Any somatic diseases which can influence the remineralization of enamel according to anamnesis;

6. Pregnancy or breast feeding according to anamnesis;

7. Disagreement to follow protocol's procedures - regular cleaning of teeth with tested products etc.

\section{Toothpaste}

Toothpaste "BIOMED WHITE COMPLEX/CHARCOAL» which contains a mix of charcoal, activated coal, and bamboo's coal was used during the trial.

\section{Ethics}

The trial was performed according to Declaration of Helsinki and Good Clinical Practice (ICH GCP).

All subjects before inclusion had been informed on the study design and procedures and signed the informed consent form.

\section{Statistical analysis}

SPSS statistical software for Windows was used for the statistical analysis of data. All results were presented as the Mean \pm SD of the measurements. The data were analyzed by the paired Student's test for percentage of $\mathrm{pH}$ variation using a $5 \%$ threshold for statistical significance. The level of significance was set at 0.05 .

\section{Results}

In total 60 volunteers at the age of $19-25$ participated in the research (Table 1). Assessment of dental plaque was performed on Visit 1 before and after teeth cleaning for 2 minutes, on Visits 2 and 3. Hygiene index on these visits are shown in (Table 2). The amount of dental plaque immediately after brushing became lower by $17.2 \%$, after 28 days the index lowered by $30 \%$ and on Visit 3 after 56 days 
Table 4: Assessment of tooth color and whitening efficiency Mean $( \pm S D)$.

\begin{tabular}{|c|c|c|c|}
\hline Index & Visit 1 & Visit 2 & Visit 3 \\
\hline On the scale Vita Classic & $4( \pm 0)$ & $3.36( \pm 0.23)$ & $3.36( \pm 0.23)$ \\
\hline
\end{tabular}

Table 5: Evaluation of enamel resistance.

\begin{tabular}{|c|c|c|c|}
\hline Index & Visit 1 & Visit 2 & Visit 3 \\
\hline $\mathrm{ER}^{\mathrm{a}}$ & $35.83( \pm 8.69)$ & $25.5( \pm 8.72)$ & $18.67( \pm 8.33)$ \\
\hline
\end{tabular}

${ }^{\mathrm{a} E R}=$ Enamel resistance

the amount of dental plaque was lower by $36 \%$ from baseline. It could be seen that cleaning effect had a mild cumulative effect (Figure 1).

The assessment teeth sensitivity was performed by two methods (Shtorina Index and Schiff test) on Visits 1, 2 and 3. Analysis was done only for a part of the selection which had initial teeth sensitivity on Visit 1 (Table 3). Insensitivity of teeth hyperesthesia after 28 days of product usage lowered by $65 \%$, and after 56 days of usage Schtorina index was lower by $91 \%$ comparing with results of Visit 1 . Amount of teeth with hyperaesthesia lowered by $65 \%$ after 28 days of use of toothpaste «BIOMED WHITE COMPLEX/CHARCOAL», after 56 days - up to $91 \%$.

Dentin sensitivity to air-cold stimuli (Schiff test) was determined by reaction of the patient on stream of compressed air, that is pointed at the tested surface (on pre-neck area from vestibular side of the teeth) the distance is near $1 \mathrm{~cm}$.

Visual Analogue Scale (VAS) was used for self-assessment of the pain levels on probe by the patient where value from 1 to 3 was met with light pain levels, from 4 to 6 points - middle, and value 7 and higher-severe (Figure 2). As a result of the study the pain intensity decreased by $83 \%$ after 28 days of product usage, and after 56 days by $97 \%$.

Also evaluation of impact of tested toothpaste on teeth color was made. Teeth color assessment and toothpaste whitening effectiveness was done by method of comparing results on Vita Classic scale on visits 1 and 3 (Table 4).

Whitening up to 1 tone occurred in $28,33 \%(\mathrm{n}=17)$ of the volunteers, up to 0,5 tone-in $71,67 \%(n=43)$ of the volunteers.

The assessment of enamel remineralization was conducted using enamel resistance test on Visits 1 and 3. The results shown below reflect significant improvement of enamel condition (Table 5). Resistibility of enamel to acid grew up to $28,84 \%$ and $47,91 \%$ after 1 and 2 months of usage according in comparison with baseline, Visit 1. Anti-caries effect was determined by Decay-Missing-Filled Index (DMF) comparing results on Visits 1 and 3. During the trial index did not change for any of the patients and no new or developing caries were detected. The results represent anti-caries effect of tested product. During the study no side effects were observed on any of included volunteers.

\section{Discussion}

Toothpaste «BIOMED WHITE COMPLEX/CHARCOAL» showed good cleaning properties after first usage - hygiene index lowered by $17,2 \%$ after the first usage. Besides that, the effect was cumulative and it was observed that forming of dental plague lowered and hygiene index was lower than on the baseline by $30,6 \%$
Table 6: Assessment of teeth sensitivity.

\begin{tabular}{|c|c|c|c|}
\hline & GII $^{\mathbf{a}}$ & Number of teeth with hyperesthesia & ACSDS $^{\mathbf{b}}$ \\
\hline Visit 2 & $-65,68 \%$ & $-65,22 \%$ & $-83,96 \%$ \\
\hline Visit 3 & $-91,35 \%$ & $-91,30 \%$ & $-97,11 \%$ \\
\hline
\end{tabular}

${ }^{\mathrm{a}} \mathrm{GII}=$ Gipertensia Intensity Index (Shtorina index) ${ }^{\mathrm{b}}$ ACSDS = Air-Cold Stimulus Dentin Sensitivity (Shiff test)

and $35,98 \%$ after 1 and 2 months respectively. These results with considering multiple comparisons were statistically significant. Teeth sensitivity decreased substantially after 1 and 2 months as shown in table 6. Using three methods of evaluation tested product proved strong positive influence on teeth sensitivity.

These results were also statistically significant considering multiple comparisons. Whitening effect was moderate and appeared after 1 month of usage. The effect rated from half tone to 1 tone. The result was statically significant considering multiple comparisons. One tone whitening happened with $28,33 \%(\mathrm{n}=17)$ of volunteers and up to 0,5 of tone-for $71,67 \%(n=43)$ of the volunteers. Thus the whole sample had a moderate whitening effect from half of tone to one tone.

Remineralization and indirect anti-caries effect of the toothpaste were tested with Enamel Resistance Test (TER) on Visits 1, 2, and 3. Resistibility of enamel to acid grew up to $28,84 \%$ and $47,91 \%$ after 1 and 2 months of use respectively. It is obvious that toothpaste «BIOMED WHITE COMPLEX/CHARCOAL» has excellent remineralization effect. These results were statistically significant with multiple comparisons.

During the study no changes in DMF index were observed in any of the volunteers. This allows us to state that forming of caries decreased in a short time period ( 2 month) due to tested product usage.

\section{Conclusion}

In conclusion, cleaning and whitening effect of toothpaste «BIOMED CHARCOAL WHITE» is clinically proven by results of the research, As well as anti-hypersensitivity effect and remineralization properties shown in the article.

\section{Role of the Funding Source}

Financial support of the study was provided by company LLC «Rusexport », Russia.

\section{References}

1. Newsom SW. Hygiene and the ancient Romans. Br J Infect Control. 2004; 5: $25-27$.

2. Juurlink DN. Activated charcoal for acute overdose: A reappraisal. Br J Clin Pharmacol. 2016; 81: 482-487.

3. Karonidis A, Delikonstantinou I, Tsoutsos D. Use of Actisorb* dressings over a skin-grafted infected wound. Burns. 2011; 37: 360-361.

4. Chakravarthi A, Srinivas CR, Mathew AC. Activated charcoal and baking soda to reduce odor associated with extensive blistering disorders. Indian J Dermatol Venereol Leprol. 2008; 74: 122-124.

5. Srinivas SR, Daniel DD, Kalyan CG. Oral health: Charcoal brushes. British Dental Journal. 2014; 217: 3-3.

6. Garza LA, Thompson G, Cho SH, Berzins DW. Effect of tooth brushing on shade and surface roughness of extrinsically stained pressable ceramics. The Journal of Prosthetic Dentistry. 2016; 115: 489-494. 